\title{
Scanning Electron Microscopic Investigations to Clarify the Role Played by the Endocardium in the Origin of Dilated Cardiomyopathy(DCM)
}

\author{
Masoud Mirzaie', Michael Schultz ${ }^{2}$, Peter Schwartz ${ }^{2}$, Zaur Guliyev ${ }^{1}$ and Sheila Fatehpur ${ }^{1}$ \\ ${ }^{1}$ Department of Vascular Surgery, Lippe-Lemgo Hospital, University clinics OWL, Germany \\ ${ }^{2}$ Institute for Anatomy, University of Göttingen, Göttingen, Germany \\ *Corresponding author: Masoud Mirzaie, Head of the Department of Vascular Surgery University Hospital of Lemgo Rintelner Straße
} 85, 32657 Lemgo Rintelner Str. 8532657 Lemgo Germany, Germany

\begin{abstract}
Dilated cardiomyopathy (DCM) is a cardiac disease characterized by dilatation and impaired systolic function of the left or both ventricles. The etiology of DCM is multifactorial, and many different clinical conditions can lead to the phenotype of DCM. During recent years, the pathophysiology of DCM has been under intensive investigation, and, thereby, the knowledge of DCM has increased rapidly. However, the pathophysiological mechanisms, by which morphological modifications eventually result in clinical heart failure, are complex and not yet totally resolved. Better knowledge of the morphological background and disease-originating mechanisms would probably help us to focus early treatment on the right subjects and potentially also develop new treatment options in the affected patients. This study aimed to investigate the pathophysiological origin of DCM from a morphological point of view. Therefore, scanning electron and polarised light microscopic investigations on explanted hearts from DCM patients were carried out to determine the morphology of the endocardium. Tissue samples were taken from 4 male (average age; 72.21/years) and 2 female DCM patients (63.14years). The study population included patients suffering from DCM who were listed on transplant waiting lists while being clinically categorized as stage NYHA III-IV. Patients' hearts were explanted for cardiac transplantation and the explanted hearts were examined by scanning electron microscopy and polarised light microscopic investigations. The endocardial layer was partially desquamated from the basement membrane and showed isolated island-like cell formations. Areas of loosened cells connected to each other and to the basement membrane, abrasion of the endothelial cells, formation of filiform and lamellar Lambl's excrescences, locally well-defined elevations above the intact endothelium, calcium deposits and hyperplasia of collagen fibers were detected. There were also formations resembling fungal micelles.
\end{abstract}

Keywords: DCM; Electron Microscopy; Thoracic aortic aneurysm; Electron microscopy

\section{Introduction}

Dilated cardiomyopathy (DCM) is a cardiac disease characterized by dilatation and impaired systolic function of the left or both ventricles. DCM causes considerable morbidity and mortality. The etiology of DCM is multifactorial, and many different clinical conditions can lead to the phenotype of DCM. During recent years it has become evident that genetic factors play an important role in the etiology and pathogenesis of idiopathic DCM [1]. The pathophysiology of DCM has been under intensive investigation, and, thereby, the knowledge of DCM has increased rapidly. The genetic background of the disease seems to be relatively heterogeneous, and the disease-associated mutations affect mostly whole families and only a few separate patients. Disease-associated mutations have been detected for example, in genes encoding sarcomere, cytoskeletal, and nuclear proteins, as well as proteins involved with regulation of $\mathrm{Ca} 2+$ metabolism. However, the pathophysiological mechanism, by which morphological mutations eventually result in clinical heart failure, are complex and not yet totally resolved 
[2-5]. Better knowledge of the pathophysiological background and disease-originating mechanisms would probably help us to focus early treatment on the the most acute subjects and potentially also develop new treatment modalities and improve cardiac outcome in the affected patients.

Most of the trials investigating the origin of DCM are focused on genetic disorders. However, in addition to genetic and pathophysiological investigations, morphological analyses of the internal structure of the heart are becoming increasingly important and may contribute to an explanation of the origin of the occurrence of DCM $[6,7]$. It is well known that genetic factors play an important role in the etiology and pathogenesis of idiopathic DCM [2, 8-10]. The origin of DCM as an endocardium-based disease has, so far, never been described. For this study, we investigated and now describe the morphology of the endocardium of DCM patients using polarised light microscopy in addition to conventional scanning electron microscopical techniques. Especially, we were interested in identifying a potential origin of DCM.

\section{Materials and Methods Tissue Samples}

Tissue samples from explanted hearts were taken from DCM patients undergoing routine cardiac surgery (cardiac transplantation) at the Department of Thoracic, Heart and Vascular Surgery, University Hospital of Goettingen, Germany. The biopsies were taken from 4 male (average age; $72+2$ years) and 2 female DCM patients $(63+1$ years). The study population included cardiac transplant patients suffering from endstage DCM (NYHA III-IV). Table 1 presents summarized data of the basic patient characteristics.

Table 1: Baseline characteristics of the study population.

\begin{tabular}{|c|c|c|}
\hline & \multicolumn{2}{|c|}{ Dilatative Cardiomyopathy $(n=6)$} \\
\hline & Male & Female \\
\hline Age & $4(72,1$ years $)$ & 2 (72.14 years) \\
\hline \multicolumn{3}{|c|}{ Comorbidities } \\
\hline Nicotin, active & $3(75 \%)$ & $1(50 \%)$ \\
\hline Ex-nicotin & $1(25 \%)$ & 0 \\
\hline Hypertension & $4(100 \%)$ & $1(50 \%)$ \\
\hline Diabetes & $1(25 \%)$ & $1(50 \%)$ \\
\hline Hyperlipoproteinaemia & $2(50.0 \%)$ & 0 \\
\hline Hyperuricaemia & $1(25 \%)$ & 0 \\
\hline CHD & $75(50 \%)$ & $1(50 \%)$ \\
\hline Carcinoma & 0 & 0 \\
\hline Renal insufficiency & $2(50 \%)$ & 0 \\
\hline COPD, emphysema & $1(25 \%)$ & $1(50 \%)$ \\
\hline
\end{tabular}

Continuous variables are presented as mean + standard deviation. Categorical variables are presented as an absolute percentage. Abbreviations: CHD: Coronary Heart Disease, COPD: Chronic obstructive pulmonary disease

\section{Scanning electron microscopy}

In order to reveal the morphology of the explanted hearts, scanning electron microscopy was performed in all tissue samples. Specimens taken from the endocardium were fixed for 6 hours in a solution containing $2.5 \%$ glutaraldehyde and $0.2 \mathrm{~m} \mathrm{Mol} \mathrm{cacodylate.}$ Afterwards, samples were dehydrated in a series of increasing concentrations of alcohol. After critical point drying, all samples were sputtered with gold-palladium. Samples were visualized using the digital scanning microscope (Zeiss DSM 960, Germany).

\section{Results}

\section{Scanning electron microscopical findings}

In all the tissue materials investigated, the endothelial cells were mostly desquamated. The remaining endothelial cells showed a loose binding to each other. At higher magnification, the endothelial cells appeared swollen and took on a foamlike appearance. The endothelial cells also showed similar metaplasia and loose binding to each other as in other endocardium parts. In addition, we found diffusely distributed sites of columnar endothelial cell formations in all tissues, some of which had lost their intercellular junction (Figures. 1,2). In such islets the endothelial cells had, in places, agglomerated to form columnar structures (Figure 3). These structures obviously represent a transitional form to Lambl's excrescences. The Lambl excrescences occurred in a filiform and a lamellar form. Often the excrescences did not have an endothelial coating (Figure 4). Hyperplasia of the collagen fibers was clearly visible in such excrescences in different layers. The ratio of filiform to lamellar forms was approximately $80 \%$ in all specimens. Very often, crater-like „punched“ defects were visible on the surface of the myocardium, which showed an endothelial coating at higher magnification (Figure 5). On such altered endothelial cells, micellelike structures were visible (Figure 6). At higher magnification the micelle-like structures are better visible (Figure 7). 


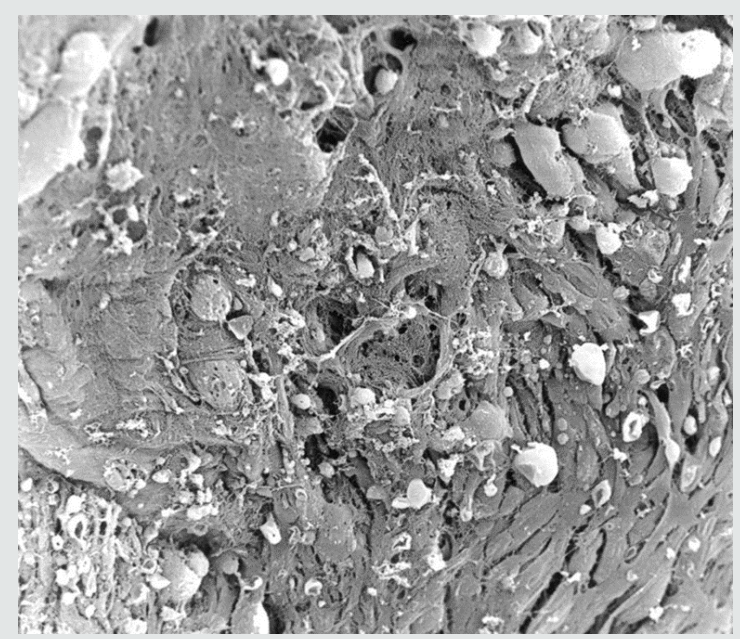

Figure 1: Electron microscopical view of endocardium of explanted hearts with DCM, 500 x magnification: desquamation of the endothelial cells with a loose binding to each other.

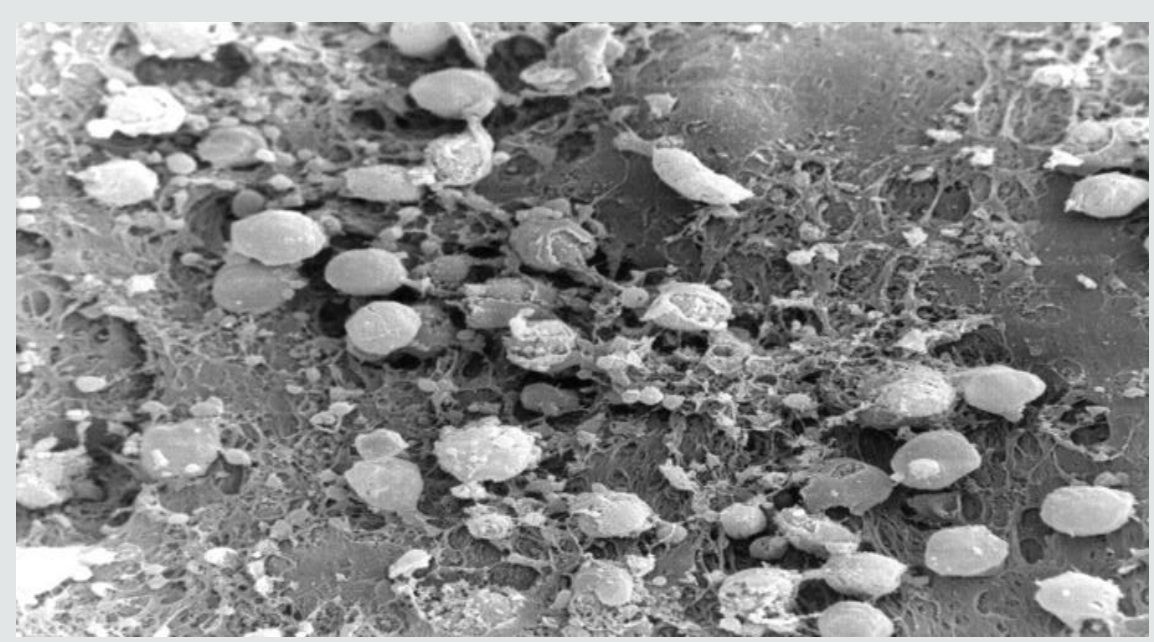

Figure 2: Electron microscopical view of endocardium of Hearts with DCM, 1000 x magnification: at higher magnification, the endothelial cells appeared swollen with a foamlike appearance.

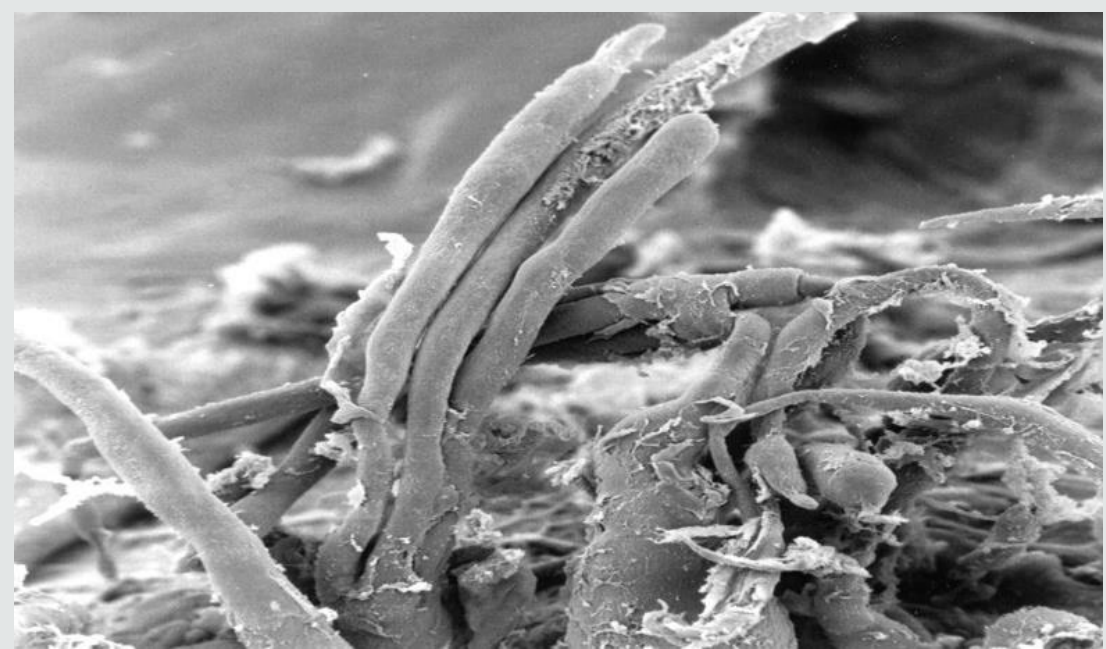

Figure 3: Electron microscopical view of endocardium of heart with DCM, 200 x magnification: filiform Lambl's excrescences. 


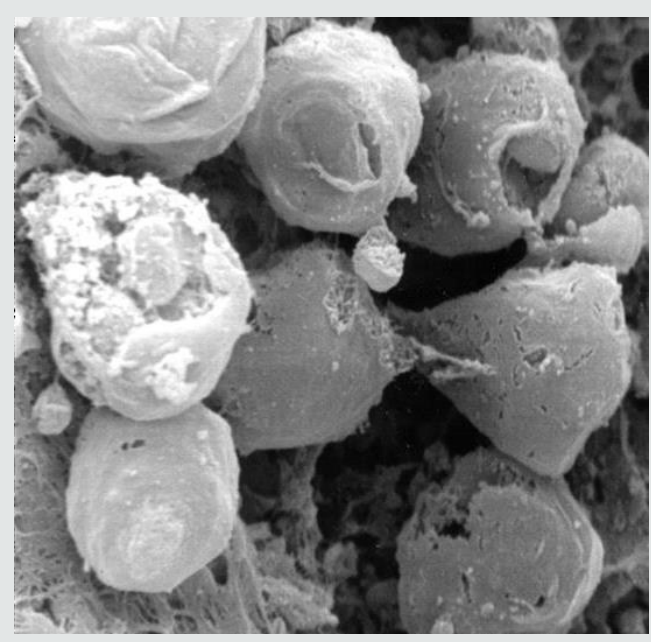

Figure 4: Electron microscopical view of endocardium of heart with DCM, 3000 x magnification: dendothelial desquamation on Lambl's excrescences.

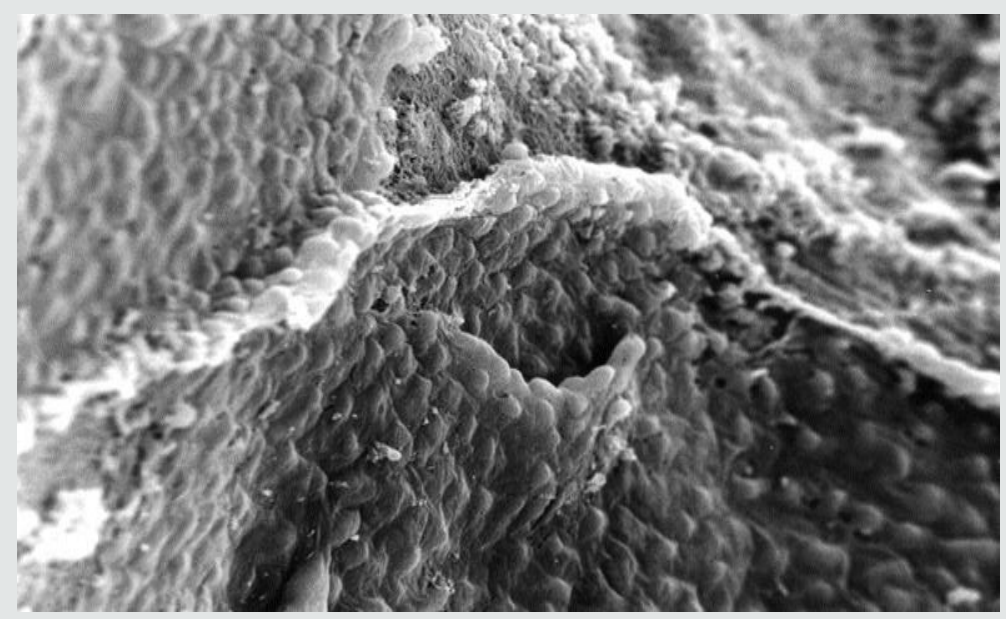

Figure 5: Electron microscopical view of endocardium of heart with DCM, $330 \times$ magnification: crater-like „, punched“ defects.

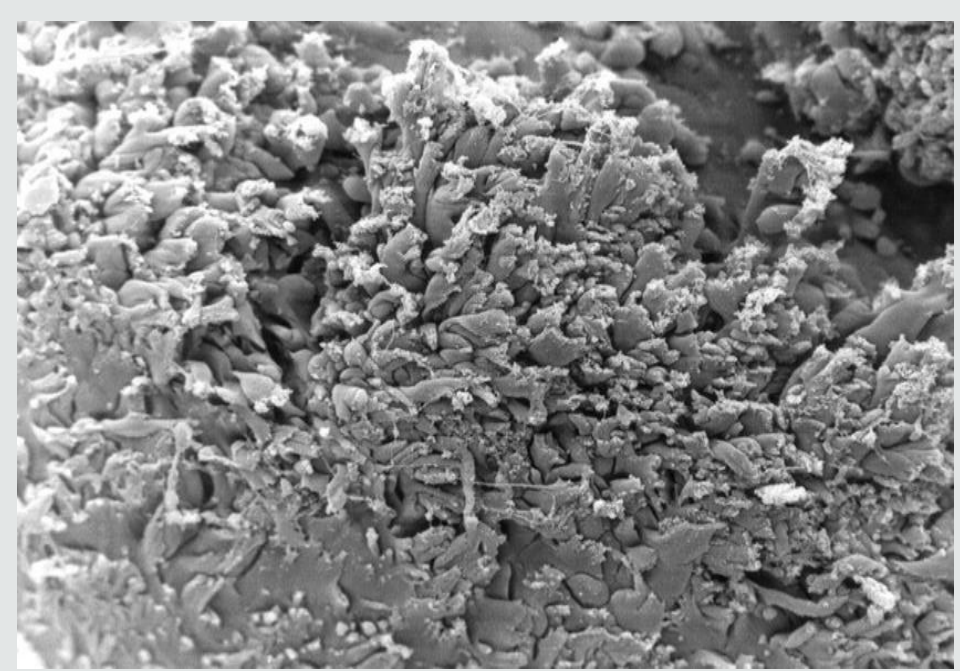

Figure 6: Electron microscopical view of endocardium of heart with DCM, $200 \mathrm{x}$ magnification: micelle-like structures. 


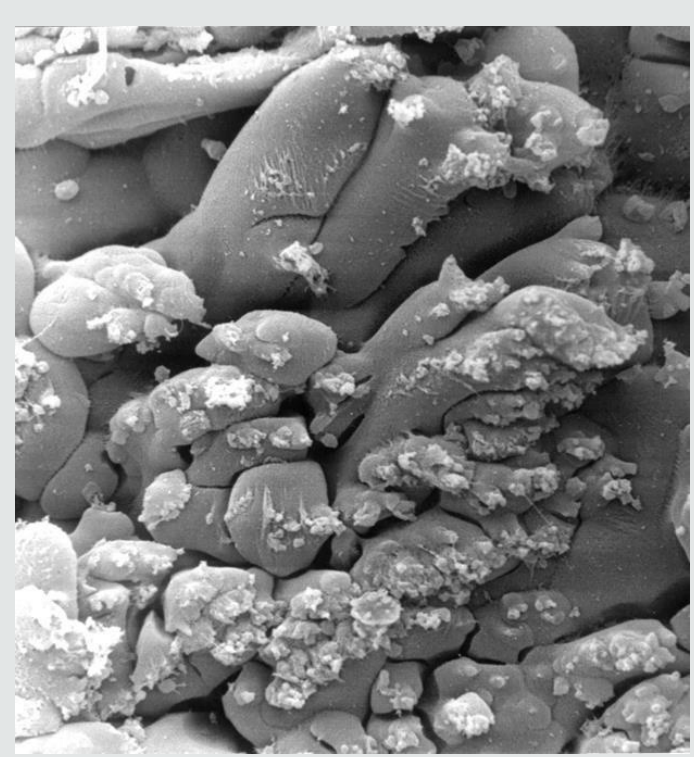

Figure 7: Electron microscopical view of endocardium of heart with DCM, 1000 x magnification: higher magnification of micelle-like structures.

\section{Discussion}

Multiple etiologies of DCM, including ischemic, genetic, toxic, viral, inflammatory, autoimmune, and, last but not least, idiopathic causes are known? [11-17]. In developed countries, the incidence of DCM is 5- 10patients/100,000 (15-17) with a prevalence of 36 patients $/ 100,000$ people $[18,19]$. Future demographic changes such as an aging population and greater life expectancy are expected to increase the incidence of DCM even more. Thus, DCM will likely play a growing economic and medical role in the coming years. $[20,21]$. Pathophysiologically, DCM is characterized by dilatation and impaired systolic function of the left or both ventricles. So far, the origin of this disease is thought to be a disorder of the cardiac muscle in which myocyte weakness leads to ventricular dilatation and heart failure $[22,23]$. However, until now, it has not yet been considered that the origin of DCM may be endocardium-based. Therefore, we performed the presented study to investigate the morphological role of the endocardium and its role in the origin of the development of DCM. In the myocardium of DCM, we were able to detect two differing findings. On the one hand, there are changes in the endothelium and the extracellular matrix, which we know in a very similar form from aortic and mitral valves explanted due to degeneration [24]. On the one hand, there is a desquamation of the endothelial cells, which lose their junctions to each other. They are also swollen. The collagen fibers are hypertrophied and have lost their helical structure. In addition, lamellar Lambl excrecences which we have been able to demonstrate in a similar form in degenerated aortic and mitral valves [24] are frequent, Craterlike defects of the endothelium have rarely been observed. On the other hand, we were able to detect fungal micelle-like strictures in all examined tissues, which mainly occurred at the edge of severely destroyed mycardium. The increased activation of matrix metalloproteinases in pathologically altered human endocardium emphasizes the crucial role of the extracellular matrix in the development of this disease [25-29]. The detection of involvement of the endocardium tissue in DCM suggests that pathophysiological processes similar to the degeneration of heart valves play a major etiological role in the development of DCM. Parallel to assumed pathophysiological processes of the heart valves, collagen substance transition disorders based on endothelial dysfunction could lead to pathologically increased stress on the extracellular matrix, which causes a similar response of the endothelium and the extracellular matrix as in the degeneration of aortic and mitral valve disease $[30,31]$. Further investigations are necessary to clarify the role of a possible infection aetiology.

\section{Conclusion}

In this study of explanted hearts of DCM patients, several morphological modifications of the endocardium and extracellular matrix are similarity of these alterations to the degeneration of aorta and mitral valves, which suggest that similar pathophysiological changes, such as possible disturbances in the synthesis of collagen fibres play an important etiological role in the development of DCM. The detection of micelle-like structures requires further clarification.

\section{Conflict of Interest}

I hereby declare that there were no financial or other interests in the execution and evaluation of this work. 


\section{References}

1. Dal Ferro M, Severini GM, Gigli M, Mestroni L, Sinagra G (2019) Genetics of Dilated Cardiomyopathy: Current Knowledge and Future Perspectives. In: Sinagra G, Merlo M, Pinamonti B (eds) Dilated Cardiomyopathy. Springer, Cham.

2. Yoshikawa T (2011) Contribution of acquired factors to the pathogenesis of dilated cardiomyopathy The cause of dilated cardiomyopathy: Genetic or acquired? (acquired-side). Circulation Journal 75(7): 1766-1773.

3. Schultheiss HP, Fairweather DL, Caforio ALP, Escher F, Hershberger RE et al. (2019) Dilated cardiomyopathy. Nat Rev Dis Primers 5(1): 32.

4. McCauley SR, Clark SD, Quest BW, Streeter RM, Oxford EM (2020) Review of canine dilated cardiomyopathy in the wake of diet-associated concerns. J Anim Sci 98(6).

5. Kinnamon DD, Morales A, Bowen DJ, Burke W, Hershberger RE et al. (2017) Toward genetics-driven early intervention in dilated cardiomyopathy: design and implementation of the DCM precision medicine study. Circ Cardiovasc Genet 10(6): e001826.

6. Wyles SP, Li X, Hrstka SC, Reyes S, Oommen S, et al. (2016) Modeling structural and functional deficiencies of RBM20 familial dilated cardiomyopathy using human induced pluripotent stem cells. Hum Mol Genet 25(2): 254-265.

7. Cowan JR, Kinnamon DD, Morales A, Salyer L, Nickerson DA, et al. (2018) Multigenic disease and bilineal inheritance in dilated cardiomyopathy is illustrated in non-segregating LMNA pedigrees. Circ Genom Precis Med 11(7): e002038.

8. Wilsbacher LD (2020) Clinical Implications of the Genetic Architecture of Dilated Cardiomyopathy. Curr Cardiol Rep 22(12): 170.

9. Marian AJ, van Rooij E, Roberts R (2017) Genetics and genomics of singlegene cardiovascular diseases: Common hereditary cardiomyopathies as prototypes of single-gene disorders. J Am Coll Cardiol 68(25): 28312849

10. Pasipoularides A (2019) Morphomechanic phenotypic variability of sarcomeric cardiomyopathies: a multifactorial polygenic perspective. J Mol Cell Cardiol 126: 23-35.

11. Tschöpe C, Ammirati E, Bozkurt B, Caforio ALP, Leslie T, et al. (2020) Myocarditis and inflammatory cardiomyopathy: current evidence and future directions. Nat Rev Cardiol 12: 1-25.

12. Heinig M, Adriaens ME, Schafer S, van Deutekom HWM, Lodder EM, et al. (2017) Natural genetic variation of the cardiac transcriptome in nondiseased donors and patients with dilated cardiomyopathy. Genome Biol 18: 170 .

13. Frantz S, I Falcao-Pires I, Balligand JL, Bauersachs J, Dirk Brutsaert D, et al. (2018) The innate immune system in chronic cardiomyopathy: a European Society of Cardiology (ESC) scientific statement from the Working Group on Myocardial Function of the ESC. Eur J Heart Fail 20(3): 445-459.

14. Bracamonte-Baran W, Čiháková D (2018) Cardiac autoimmunity: myocarditis. Adv Exp Med Biol 1003: 187-221.

15. Hänselmann A, Veltmann C, Bauersachs J, Berliner D (2020) Dilated cardiomyopathies and non- compaction cardiomyopathy. Herz 45(3): 212-220.
16. Fairweather DL, Cooper LT, Blauwet LA (2013) Sex and gender differences in myocarditis and dilated cardiomyopathy. Curr Probl Cardiol 38(1): 7-46

17. Cassani M, Fernandes S, Vrbsky J, Ergir E, Cavalieri F, et al. (2020) Combining Nanomaterials and developmental pathways to design new treatments for cardiac regeneration: the pulsing heart of aAdvanced therapies. Front Bioeng Biotechnol 8: 323.

18. Tripathi R, Sullivan R, Fan THM Wang D, Sun Y, Reed GL, et al. (2017) Enhanced heart failure, mortality and renin activation in female mice with experimental dilated cardiomyopathy. PLoS One 12(12): e0189315.

19. Tabish AM, Azzimato V, Alexiadis A, Buyandelger B, Knöll R (2017) Genetic epidemiology of titin- truncating variants in the etiology of dilated cardiomyopathy. Biophys Rev 9(3): 207-223.

20. Knapp M, Wong G (2020) Economics, and mental health: the current scenario. World Psychiatry 19(1): 3-14.

21. Jatain S, Kapoor A, Sinha A, Khanna R, Kumar, et al. (2016) Metabolic manipulation in dilated cardiomyopathy: Assessing the role of trimetazidine. Indian Heart J 68(6): 803-808.

22. Law ML, Cohen H, Martin AA, Angulski ABB, Metzger JM (2020) Dysregulation of Calcium Handling in Duchenne Muscular DystrophyAssociated Dilated Cardiomyopathy: Mechanisms and Experimental Therapeutic Strategies. J Clin Med 9(2): 520.

23. Leybaert L, Lampe PD, Dhein S, Kwak BR, P Ferdinandy P, et al. (2017) Connexins in Cardiovascular and Neurovascular Health and Disease: Pharmacological Implications. Pharmacol Rev 69(4): 396-478.

24. Mirzaie M, Schultz M, Schwartz P, Coulibaly M, Schondube F (2003) Evidence of woven bone formation in heart valve disease. Ann Thorac Cardiovasc Surg 9(3): 163-169.

25. Torregrosa-Carrión R, Luna-Zurita L, García-Marqués F, D’Amato G, Piñeiro-Sabarís R, et al. (2019) NOTCH Activation Promotes Valve Formation by Regulating the Endocardial Secretome. Mol Cell Proteomics 18(9): 1782-1795.

26. Rydeen AB, Waxman JS (2016) Cyp26 Enzymes Facilitate Second Heart Field Progenitor Addition and Maintenance of Ventricular Integrity. PLoS Biol 14(11): e2000504.

27. Kovacic JC, Dimmeler S, Harvey RP, Finkel T, Aikawa E, et al. (2019) Endothelial to Mesenchymal Transition in Cardiovascular Disease. J Am Coll Cardiol 73(2): 190-209.

28. Foulquier S, Daskalopoulos EP, Lluri G, Hermans KCM, Deb A, et al. (2018) WNT Signaling in Cardiac and Vascular Disease. Pharmacol Rev $70(1): 68-141$.

29. Zhang J, Tao R, Campbell KF, Carvalho JL, Ruiz EC, et al. (2019) Functional cardiac fibroblasts derived from human pluripotent stem cells via second heart field progenitors. Nat Commun 10: 2238.

30. TayalU, Wage R, Ferreira PF, Nielles-Vallespin S, Epstein FH, et al. (2019) The feasibility of a novel limited field of view spiral cine DENSE sequence to assess myocardial strain in dilated cardiomyopathy. Magma 32(3): 317-329.

31. Jaber WA, Maniu C, Krysiak J, Shapiro BP, Meyer DM, et al. (2009) Titin Isoforms, Extracellular Matrix and Global Chamber Remodeling in Experimental Dilated Cardiomyopathy: Functional Implications and Mechanistic Insights. Circ Heart Fail 1(3): 192-199. 
This work is licensed under Creative Commons Attribution 4.0 License

To Submit Your Article Click Here:

Submit Article

DOI: 10.32474/ACR.2020.03.000161

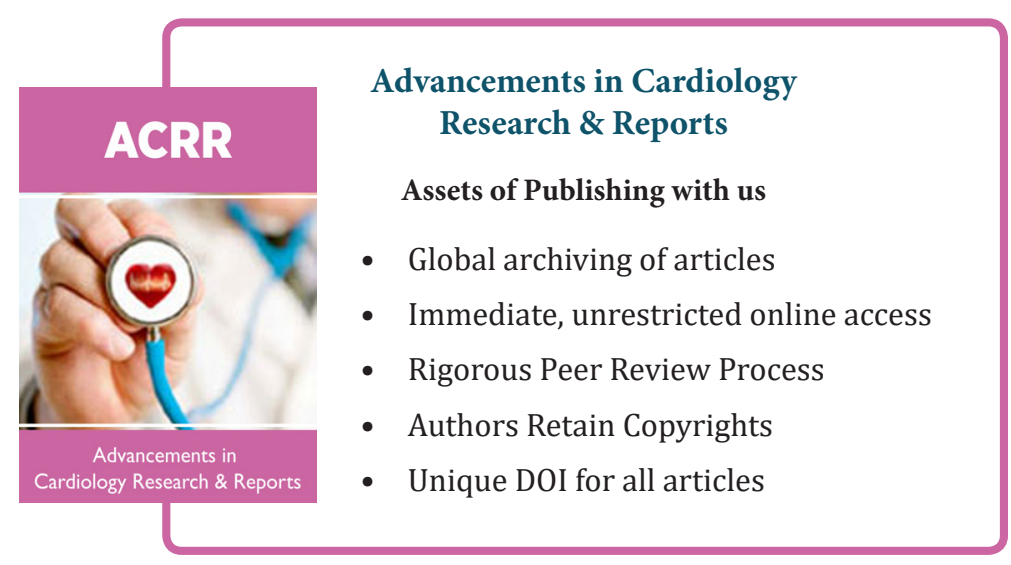

